Français, anglais et allemand: trois langues rivales entre 1850 et 1945. French, English and German: three languages in competition between 1850 and 1945

\title{
Institutionnalisation et concurrence.La langue française et anglaise dans les écoles secondaires en Allemagne(1837-1945)
}

\section{Marcus Reinfried}

\section{(2) OpenEdition}

Journals

\section{Édition électronique}

URL : https://journals.openedition.org/dhfles/4078

DOI : $10.4000 /$ dhfles.4078

ISSN : 2221-4038

\section{Éditeur}

Société Internationale pour l'Histoire du Français Langue Étrangère ou Seconde

\section{Édition imprimée}

Date de publication : 1 décembre 2014

Pagination : 11-32

ISSN : 0992-7654

Référence électronique

Marcus Reinfried, «Institutionnalisation et concurrence.La langue française et anglaise dans les écoles secondaires en Allemagne(1837-1945) ", Documents pour l'histoire du français langue étrangère ou seconde [En ligne], 53 | 2014, mis en ligne le 08 septembre 2017, consulté le 28 mai 2021. URL: http://journals.openedition.org/dhfles/4078; DOI : https://doi.org/10.4000/dhfles.4078

Ce document a été généré automatiquement le 28 mai 2021

(c) SIHFLES 


\title{
Institutionnalisation et concurrence.La langue française et anglaise dans les écoles secondaires en Allemagne(1837-1945)
}

\author{
Marcus Reinfried
}

\section{L'introduction du français et de l'anglais dans les Gymnasien et Realschulen}

Le sociologue Norbert Elias a analysé dans sa monographie Über den Prozeß der Zivilisation (parue en français sous le titre La civilisation des mœurs) l'utilisation des notions de culture et de civilisation en Allemagne (Elias $\left.{ }^{1} 1939 / 1997: 89-131\right)$. Vers la fin du siècle des Lumières, ces deux mots constituaient une polarité conceptuelle. L'idée de « civilisation » était pour Immanuel Kant (comme pour beaucoup d'autres intellectuels allemands de l'époque) surtout liée au progrès technique d'une part et d'autre part aux nobles ainsi qu'à la société courtoise qui, en Allemagne, tenait la «bourgeoisie cultivée » (Bildungsbürgertum) à distance ${ }^{1}$. La notion de culture, par contre, liée à une éthique du comportement humain, était attribuée par la «bourgeoisie cultivée » à ellemême. À la fin des Lumières, cette couche de la société l'emporta idéologiquement sur la bourgeoisie commerciale et technique (encore relativement limitée à l'époque à cause du commencement très tardif de la révolution industrielle en Allemagne). C'est donc bien la «bourgeoisie cultivée » qui, à l'époque de la philosophie idéaliste de Kant et de Fichte et de la littérature classique de Goethe et de Schiller, définit les objectifs majeurs des écoles secondaires qu'elle imprègne de son habitus spécifique. C'était l'habitus d'une classe moyenne ambitieuse, élitiste, fière de ses capacités intellectuelles, soulignant l'importance de la rigueur, de l'intériorisation du savoir et du développement de la personnalité et méprisant une certaine superficialité qu'elle percevait souvent chez les nobles et les commerçants. Au tournant du siècle, cette 
classe sociale s'intéresse, pour son immense majorité, à l'Antiquité grecque, essentiellement au théâtre, à la sculpture et à la philosophie. Un renouvellement de l'humanisme s'annonce. Ce courant néo-humaniste (qui s'est développé aussi dans d'autres pays européens) sera très fort en Allemagne, même plus fort qu'en Angleterre, en France ou en Italie où il s'est aussi profondément ancré dans les universités.

2 En 1787, la Prusse créa un Oberschulkollegium, une administration suprême et indépendante soumise seulement au roi, pour permettre le contrôle des écoles par l'État (Jeismann 1974 : 26-27). La tâche principale de cette autorité était d'initier une uniformisation des écoles latines. À partir de 1808, on fonda une section de l'enseignement scolaire au Ministère de l'intérieur de Prusse dont le directeur fut temporairement Wilhelm von Humboldt, le grand philosophe du langage (ibid.: 295-334). Les défaites militaires de l'armée prussienne dans les guerres napoléoniennes ayant contribué à ce que le gouvernement royal soit plus déterminé que jamais à mettre en œuvre certaines réformes administratives, le Gymnasium (le lycée classique allemand) fut définitivement complété par l'institutionnalisation strictement réglementée de l'Abitur $(\mathrm{du} \mathrm{bac})^{2}$ et on instaura l'obligation de passer un examen universitaire spécifique pour les candidats à un poste d'enseignant dans le secondaire (Haenicke 1982: 32-33). On établit donc un contrôle de qualité de l'enseignement exercé par l'État et on modernisa la structure de l'enseignement. Ces réformes institutionnelles allaient de pair avec l'idée pédagogique de la allgemeine Menschenbildung (éducation humaine générale) qui comprenait un large développement des capacités intellectuelles et valorisait surtout l'étude approfondie des langues classiques et la lecture de textes littéraires; les objectifs pédagogiques d'affûter la pensée des élèves et de développer leur imagination étaient censés encourager aussi le développement de leur sens éthique (Jeismann 1974 : 324-331; Hüllen 2005 : 81-83).

3 L'éducation humaniste a encore gagné en prestige au cours de la première moitié du $\mathrm{XIX}^{\mathrm{e}}$ siècle en Allemagne et est devenue la figure de proue d'une grande partie de la bourgeoisie cultivée. À partir de 1837, on apprenait le latin dans les lycées en moyenne neuf heures et demie par semaine dans chaque classe (Christ \& Rang 1985, VII : 23, 27). À la fin du XIX ${ }^{e}$ siècle et au début du $\mathrm{XX}^{\mathrm{e}}$ siècle, on en était encore à environ sept heures (ibid.: 41, 47). Le grec ancien, de son côté, a profité aussi tout au long du XIX ${ }^{\mathrm{e}}$ siècle d'un horaire avantageux avec en moyenne six heures hebdomadaires (ibid.: 23, 27, 41). Grosso modo, environ $40 \%$ de l'ensemble du temps d'enseignement disponible au Gymnasium étaient réservés aux langues anciennes. Il ne restait plus beaucoup de temps pour les autres matières.

4 Ce n'est donc pas étonnant de voir émerger un mouvement opposé à l'humanisme, le «réalisme » (dont les racines remontaient au philanthropisme du XVIII ${ }^{e}$ siècle). Ce mouvement n'était pas uniforme, il s'agissait plutôt d'un rassemblement de diverses tendances. Il y avait d'un côté les partisans d'une intégration plus poussée des sciences naturelles dans l'enseignement, de l'autre côté les avocats d'une prise en considération plus ample des langues et cultures modernes. Parmi les langues modernes, il n'y avait que le français à être régulièrement enseigné dans les Gymnasien en Prusse à partir de 1837 (Christ 1983 : 99) ${ }^{3}$. Mais cet enseignement ne dépassait que rarement deux heures hebdomadaires, et il se limitait encore dans toute l'Allemagne à trois ou quatre années dans la première moitié du XIX siècle ; vers la fin de ce siècle, on a augmenté le quota horaire de l'enseignement du français en ajoutant une troisième heure par semaine dans certaines classes et en prolongeant la durée de l'enseignement à sept années 
(Christ \& Rang 1985, VII : 23, 27, 41). Néanmoins, ce n'était pas suffisant pour permettre un apprentissage approfondi.

5 Par conséquent, pour combler cette lacune, on a établi dans la première moitié du XIX siècle des höhere Bürgerschulen, des écoles secondaires de cycle court pour des jeunes destinés à des professions "bourgeoises ", surtout commerciales ou techniques, donc dans des domaines éloignés des études universitaires de l'époque. Jusqu'en 1850, un tiers des élèves prussiens fréquentant une école de niveau supérieur allait dans une höhere Bürgerschule (entre 1 et $2 \%$ de la population masculine des villes), deux tiers des élèves dans un Gymnasium (entre 2 et $4 \%$ de la population masculine des villes); ces chiffres ont ensuite triplé au cours de la deuxième moitié du XIX ${ }^{\mathrm{e}}$ siècle (Lundgreen $1980: 79$; Jeismann $1974: 52$, note 22). Mais seule une fraction infime des lycéens est resté au Gymnasium jusqu'au bac (environ un quinzième au début et un cinquième à la fin du XIX ${ }^{e}$ siècle). Le certificat d'études secondaires moyennes (mittlere Reife) qu'un lycéen obtenait en général après six années et dans les höhere Bürgerschulen sept (avec un examen final) apportait déjà d'importants avantages : il pouvait réduire la durée du service militaire (de deux ou trois à un an) et donnait souvent accès à des places d'apprentissage convoitées dans les domaines commerciaux et techniques.

Dans la deuxième moitié du siècle, on a essayé de prolonger le cursus de certaines de ces höhere Bürgerschulen, appelés aussi Realschulen, afin que les élèves de ces institutions puissent y passer le bac. Mais les nouvelles qualifications ne donnaient accès qu'à un nombre restreint de cursus universitaires; ce n'est qu'en 1900, après une longue lutte entre « réalistes » et néo-humanistes, que l'égalité de principe a enfin été fixée par un arrêté de l'empereur Guillaume II (Lexis 1902 : VII-X). À l'époque s'étaient déjà établies deux formes de Realschulen de cycle long: les Realgymnasien, qui combinaient l'enseignement du latin avec celui de deux langues modernes, et les Oberrealschulen, qui se limitaient à un enseignement approfondi de deux langues modernes. Dans les Realgymnasien, on enseignait dans la plupart des cas le latin comme première langue étrangère, le français comme deuxième et l'anglais comme troisième langue. Le nombre d'heures enseignées en Prusse à partir de 1892 était le suivant : 48 heures pour le latin (pour l'ensemble de la scolarité), 31 pour le français et 18 pour l'anglais (Christ \& Rang 1985, VII : 90). Dans les Oberrealschulen, en revanche, le français occupait, avec 47 heures de cours (pour l'ensemble de la scolarité), la place du latin, et l'enseignement de l'anglais disposait de 25 heures (ibid.: 131). Mais les Realgymnasien étaient plus fréquentés que les Oberrealschulen parce que la connaissance du latin était à l'époque encore considérée comme indispensable pour la plupart des cycles d'études. Le Realgymnasium est par conséquent devenu le grand rival du Gymnasium et l'a finalement dépassé en nombre d'élèves à la fin des années 1920 (Lundgreen 1981 : 104).

$7 \mathrm{Au}$ cours du XIX et au début du $\mathrm{XX}^{\mathrm{e}}$ siècle (jusqu'au commencement de la Première Guerre mondiale), le poids et la valeur de la langue française et de la langue anglaise étaient bien définis. Il y avait une hiérarchie qui assignait en général la première position à la langue française et la seconde position à la langue anglaise, avec quelques exceptions dans le nord de l'Allemagne. Les élèves des Gymnasien apprenaient en général le français comme matière obligatoire mais secondaire, tandis que l'enseignement de l'anglais était, même au début du XX $\mathrm{XX}^{\mathrm{e}}$ siècle, encore facultatif ou réduit à quelques heures. Dans les Realgymnasien comme dans les Oberrealschulen, les cours de français disposaient presque du double des heures (pour l'ensemble de la scolarité) que les cours d'anglais. Mais tout le monde n'était pas d'accord avec cette 
répartition du temps d'apprentissage réservé aux langues modernes. Certains représentants de l'industrie et de l'économie allemande croyaient déjà à cette époque que l'anglais était plus important que le français au niveau mondial si on prenait en considération le poids industriel et économique de la Grande Bretagne et des États-Unis (Schröder 1989: 67-68; Ostermeier $2012: 186$ ).

\section{La crise de l'enseignement des langues modernes pendant la Première Guerre mondiale et leur concurrence au cours de la République de Weimar}

8 La Première Guerre mondiale amena une césure pédagogique: on vit reculer le prestige de l'enseignement des langues modernes. Dans certaines parties de la population allemande s'est répandue "une aversion contre les langues de nos ennemis », comme l'a exprimé Hasl (1916: 106). Des nationalistes ont demandé la réduction ou même la suppression de leur enseignement. Il est même arrivé que des élèves boycottent les cours de français et d'anglais (Wiese 1915: 209). L'antipathie qui s'est développée pendant la guerre contre les Français et le français, a été en général plus grande que l'aversion contre les Anglais et l'anglais.

9 Le lendemain de la guerre, le Traité de Versailles, déclarant l'Allemagne seule responsable de la guerre et lui imposant des réparations, a encore intensifié la colère de la droite allemande. Toute l'Allemagne vivait des années difficiles à cause d'une crise économique grave et d'une inflation désastreuse, quand des troupes franco-belges ont occupé le bassin de la Ruhr en janvier 1923 pour confisquer le charbon des mines parce que les réparations n'avaient pas été payées à temps. La population allemande répondit par des actes de désobéissance civile, des grèves et des émeutes qui furent réprimés par les forces d'occupation. Cela mena à des protestations dans toute l'Allemagne et nuisit profondément à l'image des Français et des Belges.

10 Au début des années vingt, il y eut des débats sur l'ordre chronologique d'introduction des langues étrangères dans les écoles de niveau supérieur. En 1921, le Landtag (parlement régional) bavarois discuta de savoir si l'anglais pourrait être introduit comme première langue moderne et si l'italien pourrait remplacer le français comme deuxième langue moderne (anon. 1921). Max Deutschbein plaida à Hildesheim, où eut lieu en novembre 1921 l'assemblée générale du Allgemeiner deutscher Realschulmännerverein, pour l'acceptation de l'anglais comme première langue vivante en avançant des arguments didactiques (l'anglais est plus proche que le français des structures grammaticales de l'allemand, son choix facilite le transfert interlangue et correspond au principe - déjà articulé par Comenius - « Du proche au lointain, du facile au difficile » (Deutschbein 1922 : 225). Mais cet exposé provoqua encore deux réponses formulées par Stölting (1922) et Collischonn (1922) défendant le français comme première langue étrangère dans la revue du Deutscher Philologenverband (de l'association professionnelle regroupant les enseignants des écoles de niveau supérieur, surtout des Gymnasien). En 1923, en revanche, après l'occupation du bassin de la Ruhr, l'atmosphère au sein de la collectivité allemande était tellement tendue que les amis du français n'osaient plus articuler ouvertement leur opinion; une grande partie de l'opinion publique les accusait de «trahison à la patrie » (Hartig 1931: 342). L'enseignement du français fut condamné publiquement, par exemple lors des manifestations du 
Reichsbürgerrat, association conservatrice qui avait été fondée pendant la révolution allemande de 1918/19 pour s'opposer aux conseils d'ouvriers et de soldats socialistes (ibid.). Même la revue du Deutscher Philologenverband, dans laquelle les défenseurs ainsi que les adversaires du français avaient pu librement exprimer leurs différends, commença à appeler au strict remplacement du français par l'anglais (Hanf 1923; Krüper 1923).

11 L'atmosphère provoquée par l'occupation du bassin de la Ruhr a abouti à des changements dans la hiérarchie des langues étrangères. Otto Boelitz (1923), le ministre de l'éducation prussien, a introduit dans un arrêté la possibilité que les proviseurs remplacent (par une demande auprès des administrations scolaires) l'enseignement du français par celui de l'anglais, procédure adoptée par la suite dans d'autres parties de l'Allemagne. Une deuxième solution, établie dans le pays de Bade en 1923, a été de laisser les élèves choisir librement entre le français et l'anglais dans les Gymnasien (Christ \& Rang 1985, I : 82, IV : 18). Une troisième possibilité, pratiquée à partir du début de l'année scolaire 1923/24 (traditionnellement après Pâques) en Bavière, a été la décision d'établir l'anglais partout comme première langue moderne et matière principale dans les Gymnasien et Realschulen (Hartig 1931 : 339). Cette disposition a aussi été prise en 1929 par la Thuringe.

12 Ces trois mesures administratives ont eu pour effet que les chiffres des élèves apprenant l'anglais comme LV1 (Langue Vivante 1) n'ont cessé d'augmenter au cours des années vingt. Les chiffres exacts ne sont pas connus pour toute l'Allemagne et il reste même incertain s'ils pourront être calculés avec précision sur la base d'informations qu'on pourrait trouver dans des archives. Mais on dispose des chiffres exacts des écoles prussiennes de niveau supérieur (de garçons et de filles) entre 1919 et 1929 pour le français LV1 et l'anglais LV1 (Engel 1927 : 768; Simon 1929: 755). Il est important de signaler qu'environ deux tiers de la population allemande vivaient en Prusse à l'époque. Les statistiques montrent clairement que les pics de croissance eurent lieu entre 1923 et 1925 et que l'allure de la courbe de croissance des écoles enseignant l'anglais LV1 c'est ensuite sensiblement ralentie; pendant l'année scolaire 1927/28, elle se limita à 1,6\% (Engel $1927: 768)^{1}$. Sur la base des données de Simon (1929 : 755), 44,5\% des écoles prussiennes ne commencèrent à enseigner l'anglais que tout à la fin des années vingt tandis que 55,5\% d'entre elles enseignaient encore le français comme première langue moderne (chiffres calculés par l'auteur).

Les sept provinces prussiennes dans lesquelles l'anglais domine sont situées plutôt au nord ou à l'est du royaume. Il s'agit du Schleswig-Holstein, d'Hanovre, de la Prusse orientale, de la Poméranie, de la province de Saxe ainsi que de la Haute et de la Basse Silésie. À l'extérieur de la Prusse, les pays suivants privilégièrent la langue anglaise : la Bavière, le Freistaat («État libre») de Saxe, les trois villes hanséatiques (Brême, Hambourg, Lübeck), les deux États du Mecklembourg ${ }^{2}$ et (à partir de 1930) de Thuringe (Engel 1927: 769, Hartig 1931: 339). Les sept autres provinces prussiennes dans lesquelles le français a (partiellement ou même complètement) gardé son importance sont en général situées plutôt à l'est ou au sud de la Prusse. Ce sont le territoire de la Sarre, la province rhénane, la Westphalie, Berlin, le Brandebourg, la Hesse-Nassau et la Prusse occidentale (Simon 1929: 754-755). À l'extérieur de la Prusse, il faut compter encore le pays de Bade et la Hesse parmi les régions francophiles où une majorité des écoles a gardé l'enseignement du français comme première langue vivante (Engel 1927 : 769). En somme, les pourcentages de l'anglais d'une part et du français d'autre part, 
comme première et principale langue étrangère moderne, sont parvenus à la fin des années vingt presque à un niveau d'égalité ; le français domine encore le classement en Prusse, mais l'anglais a déjà de l'avance dans l'ensemble des autres pays allemands.

La deuxième moitié des années vingt s'est distinguée de la première d'un point de vue économique et politique. En effet, la classe moyenne allemande avait perdu plus de $90 \%$ de son épargne à cause de l'inflation, à son apogée à la fin de l'année 1923. Mais un changement de l'ambiance générale se produisit grâce à l'amélioration de la situation économique les années suivantes, à partir de 1925. À la fin de cette année-là, l'Allemagne et la France conclurent les accords de Locarno. Les hommes politiques d'orientation démocrate s'arrangèrent avec la France, qui pratiquait, elle aussi, sa politique étrangère avec plus de doigté; seuls le Parti national-socialiste (Nationalsozialistische Deutsche Arbeiterpartei) et le Parti national du peuple allemand (Deutschnationale Volkspartei) restèrent toujours très réservés voire hostiles à l'égard de «l'ennemi héréditaire français». En 1926, l'Allemagne entra dans la Société des Nations, le précurseur de l'Organisation des Nations Unies. En mai 1927, un arrêté prussien conseilla aux enseignants de transmettre également aux élèves, dans le cadre d'une éducation politique, «la conviction que le développement de chaque peuple est favorisé par l'appartenance à une communauté globale de tous les peuples ${ }^{3}$ (Johannsen 1929: 450). La tendance vers une politique de paix se manifesta aussi dans certains efforts de vouloir réformer la « Kulturkunde » (l'éducation culturelle), plutôt nationaliste, qui était au centre des programmes scolaires prussiens de 1924/25 (Richertsche Richtlinien) en soulignant (à côté des différences) les aspects et les intérêts communs entre la France et l'Allemagne (Reinfried 1999 : 212-213).

Dans ce contexte favorable à un rapprochement des peuples français et allemand, Adolf Grimme, social-démocrate et ministre de l'éducation prussienne depuis mars 1931, décida de rétablir l'ancienne importance du français en tant que première langue vivante. Grimme avait l'intention de standardiser l'éducation du secondaire. Il était affecté par les plaintes de l'époque disant qu'une diversification exagérée des cursus de langues étrangères multiplierait les types d'école ${ }^{4}$ et rendrait les changements d'écoles difficiles pour les élèves (Bolle 1931: 161). Le ministre distingua dans son plan entre première langue obligatoire et langue principale optionnelle (renforcée - selon les différentes classes - de une à deux heures hebdomadaires supplémentaires en comparaison à l'autre langue moderne). Le français devrait obligatoirement redevenir première langue dans les Oberrealschulen et dans une partie des Realgymnasien (les établissements où la première langue était une langue moderne et non pas le latin); il serait la seule langue dans les trois classes inférieures (c'est-à-dire les trois premières classes du cycle initial). Au début du cycle moyen, l'élève pourrait choisir entre le français ou l'anglais comme langue principale (Bolle 1931 : ibid.). Cette conception a d'abord été mise en œuvre en Prusse (à partir de l'année scolaire 1932/33), mais les ministres de l'éducation d'autres pays allemands (surtout de Bavière, du Wurtemberg, du pays de Bade et de Thuringe) se mirent d'accord pour la mettre en place à leur tour ${ }^{5}$. La réalisation pratique a été prévue pour l'année scolaire 1933/34. La Saxe, les deux Mecklembourg et cinq villes indépendantes du nord sont cependant restés attachés à l'anglais comme première langue (Führ 1972 : 303, note 29). 


\section{La réforme nazie de l'enseignement des langues étrangères}

16 Fin janvier 1933, le président Hindenburg, pris dans une crise gouvernementale difficile, nomma Adolf Hitler chancelier. Ce fut l'arrivée au pouvoir des nazis. Ceux-ci modifièrent au bout de quelques mois les institutions politiques et abolirent les droits démocratiques, alors que la suppression de la structure fédérale des Länder, profondément enracinée dans l'histoire allemande, prit plus de temps; en 1935, la Bavière et le pays de Bade promulguèrent de nouveaux horaires scolaires qui continuaient à privilégier la langue française (Christ \& Rang 1985, VII : 121, 163, 196, 260). La Prusse ne changea également rien à la position du français comme première langue dans les Oberrealschulen et une partie des Realgymnasien au début du régime nazi, quoiqu'elle n'acceptât que l'anglais (et non pas le français, comme l'avait prévu optionnellement Grimme) comme langue principale dans les cycles moyen et supérieur (Christ \& Rang 1985, I: 83, IV : 31-32). Entretemps, un Ministère de l'éducation nationale fut instauré en 1934 à Berlin ${ }^{1}$ qui élabora pendant trois ans une grande réforme du système scolaire ${ }^{2}$.

17 Les deux conseillers du ministre de l'éducation qui dirigeaient la section de l'enseignement étaient Rudolf Benze et Ernst Bargheer, deux nationaux-socialistes convaincus. Ils avaient déjà représenté le Ministère de l'éducation prussien lors de la onzième réunion du Comité de l'éducation nationale des pays allemands en novembre 1933 et y avaient attiré l'attention des représentants d'autres ministères plus conservateurs en mettant en doute de façon polémique la valeur du latin (que Benze avait enseigné lui-même comme professeur dans un Realgymnasium) et surtout celle du grec ancien; les procès-verbaux (conservés aujourd'hui aux archives fédérales de Berlin-Lichterfelde) étaient confidentiels à l'époque (Reinfried 2012 : 35). Le ministre Bernhard Rust, qui avait été aussi enseignant en langues classiques jusqu'en 1927 (mais qui s'identifiait plus à cette discipline que le «moderniste " Benze), avant de devenir politicien professionnel et fonctionnaire du NSDAP, a sans doute donné à ses deux collaborateurs l'instruction de ne pas se fixer trop tôt sur une conception plus précise concernant le choix des langues étrangères, mais d'attendre la fin des discussions avec les spécialistes des autres ministères et du NSDAP. Un programme officiel des nationaux-socialistes dans le secteur particulier de l'enseignement n'existait pas encore (Nagel 2012: 47), mais Benze (1933), auteur assidu dans le domaine de la pédagogie nazie, avait déjà publié un article dans la revue nationale-socialiste Volk im Werden sur ses idées d'une réforme de l'enseignement en intégrant aussi le problème des langues.

Rust était un personnage consciencieux, disposé à intégrer dans le processus de décision une partie relativement large du spectre argumentatif de la politique éducative, au moins tout ce qui était mis en avant dans l'aile droite allemande (Nagel 2012 : 356). La question la plus controversée sur l'avenir de l'enseignement des langues étrangères ne concernait pas les langues classiques, mais l'enseignement du français. Dans les revues néo-philologiques et didactiques, il y eut un long débat sur sa valeur. Dans Die neueren Sprachen, cinq spécialistes (un ingénieur, un gestionnaire du NSDAP, un conseiller ambassadorial, un officier de la marine allemande, un armateur) communiquèrent leurs estimations concernant l'utilité des langues modernes: l'un croyait que l'anglais était la seule langue indispensable ; pour deux autres spécialistes, l'anglais et le français étaient ex æquo en première position; pour les deux derniers, 
l'anglais était en première et le français en seconde position (Reinfried 2012:35). Ces articles sont probablement censés présenter des arguments contre l'idée répandue dans les milieux nationaux-socialistes (on la trouve aussi chez Rudolf Benze) qu'une seule langue étrangère obligatoire serait en principe suffisante pour les écoles de niveau supérieur.

Dans les articles rédigés par les experts de l'enseignement des langues (ce sont à l'époque surtout des formateurs d'enseignants stagiaires, des chargés de cours en didactique dans les universités ou des éditeurs de revues didactiques), les auteurs essayèrent souvent de sauver l'enseignement du français langue étrangère. Ils savaient probablement tous que l'anglais serait à l'avenir favorisé comme première langue, mais Siebert (1935 : 287) continua quand même à défendre le français. Hübner $(1934: 167)$ et Bohlen (1934:1-2) se limitèrent à la défense d'un enseignement du français à côté de celui de l'anglais, avec une petite réduction des heures de cours; ils analysèrent l'importance des deux cultures et littératures dont ils appréciaient la complémentarité. En revanche, l'estime de la culture française par Klapper (1935: 150-151) et par Bolle (1934: 196), qui représentaient nettement la droite allemande (mais pas encore nécessairement le nazisme), est beaucoup moins répandue. Néanmoins, Klapper (1935: 158-159) crut que l'enseignement de la civilisation française était nécessaire, mais surtout à des fins " géopolitiques ", pour que les jeunes Allemands connaissent bien le pays qui continuerait d'être leur adversaire. Bolle (ibid.), de son côté, aperçut dans la culture du peuple français (comme dans d'autres "peuples romans») "une dérive dangereuse » dont « la fonction d'alerte négative sur notre jeunesse [allemande] devra être prise en compte dans une mesure bien plus forte que sa fonction éducative positive", ce qui assignerait "le français à un statut mineur dans les écoles ». Certaines formulations de Bolle font penser à de vieux clichés que le nationalisme allemand avait construits sur les Français et la France et qui ont été perpétués et même intensifiés et radicalisés sous l'influence de l'idéologie du nazisme (Reinfried 2013 : 39-40).

20 Un autre article qui fait partie de ce débat représente la pensée des nazis dans toute sa dureté. L'auteur, resté anonyme, y soutient que " une éducation nationale et raciale responsable limitera tous les objectifs et contenus scolaires au domaine de sa propre race et de son propre peuple " et que "on évitera un enseignement qui dévoie les jeunes gens de leur cercle de vie national en leur présentant trop tôt et en trop grande quantité des choses étrangères et impropres à leur nature ethnique ${ }^{4}$ " (Anon. 1934 : 173). L'importance de la fonction formative de l'enseignement de la langue française était mise en doute, également en vue de la sélection intellectuelle des élèves dans les écoles de niveau supérieur qui devait se faire plutôt par des disciplines non linguistiques, car « nous autres Allemands, nos points forts ne résident pas en premier lieu dans les domaines du style et de l'éloquence comme c'est le cas pour le juif ou l'Européen du sud $»^{5}$ (anon. $\left.1934: 174\right)$. La conclusion de cet article, qui a, semble-t-il, été rédigé par Rudolf Benze (ceci paraît probable pour des raisons de contenu et de style et aussi à cause de l'indication du lieu de l'auteur "Berlin»), est que l'anglais deviendrait sans aucun doute première langue aussi bien que langue principale dans tous les lycées modernes (anon. $1934: 175$ ).

21 À partir de 1936, de nombreux arrêtés annoncèrent des changements pour 1938. Ils prévoyaient une restructuration profonde du système scolaire. L'enseignement secondaire, qui se subdivisait en plusieurs douzaines de filières au temps de la 
République de Weimar, fut réduit à cinq types d'écoles. Les lycées classique et scientifique étaient réservés aux garçons, le lycée d'enseignement ménager, aux filles. Ensuite, un lycée destiné à l'enseignement des langues modernes pour les garçons et pour les filles était encore prévu (c'étaient deux institutions différentes puisqu'il n'y avait pas encore de coéducation) (Homeyer 1940 : A1-2 et A9). L'anglais était partout la première langue étrangère, sauf au lycée classique où il était la troisième langue après le latin et le grec ancien. Le nouveau programme de 1938 expliqua la prépondérance de cette langue étrangère au lycée moderne (appelé désormais Oberschule) par l'affirmation qu'il s'agissait «de la langue d'un peuple dont la race nous est parente, d'un peuple ayant accompli, grâce à son grand talent politique, de hautes performances à l'échelle mondiale et ayant fait de sa langue la langue véhiculaire de l'économie mondiale » (Reichs- und Preußisches Ministerium 1938 : 208).

Le latin était en général la deuxième langue étrangère, sauf dans les lycées classiques où il était, bien sûr, en première position, et dans les écoles d'enseignement ménager qui se limitaient à l'anglais comme seule langue étrangère (Reichs- und Preußisches Ministerium 1938 : 23-30 ; Homeyer 1942: A10-A13, A13b). Le français disparaissait presque complètement, au grand dam de ses nombreux enseignants et enseignantes qui n'osaient pas critiquer ouvertement cette décision du régime nazi. La langue française survivait encore comme deuxième ou troisième langue étrangère dans les écoles de filles et comme troisième langue facultative dans la filière des langues destinée aux garçons où elle était en concurrence avec l'espagnol et l'italien. L'ensemble de ses heures d'enseignement se limitait dans ces cas-là à neuf, tout au plus à onze heures pour toute la scolarité (Homeyer 1942 : A10-A13, A13b). La dégradation de l'importance accordée au français a souvent mené à une perte de motivation chez les élèves qui continuaient à l'apprendre (Gräfer 1939 : 231).

Tandis que le nouveau programme réservait 24 pages à l'enseignement de l'anglais, il n'en consacra qu'une demie aux «langues tertiaires » français, espagnol et italien (Reichs- und Preußisches Ministerium 1938 : 207-230). Avant de décrire précisément des objectifs d'apprentissage, l'administration scolaire voulut attendre les rapports d'expériences dans l'enseignement de l'anglais et du latin sur lesquels l'enseignement des langues romanes devrait se baser systématiquement; mais les premiers "groupes de travail» (Arbeitsgemeinschaften) pour le français, l'espagnol et l'italien étaient seulement prévus pour l'année scolaire 1942/43 (Sanftleben 1938 : 164). Il n'y a rien eu de complémentaire publié à ce sujet dans les programmes scolaires, sans doute en raison des grandes difficultés que connut l'Allemagne dans la deuxième moitié de la guerre: le manque d'enseignants (qui furent de plus en plus mobilisés dans l'armée allemande) provoqua des difficultés pour assurer la permanence de l'enseignement, et l'administration scolaire donna l'instruction d'annuler plutôt l'enseignement des langues étrangères que l'enseignement de l'allemand, des mathématiques ou des sciences naturelles (Lehberger 1986: 197-198). En outre, le déroulement régulier de l'enseignement fut tellement entravé dans la phase finale de la guerre par les fréquentes alarmes causées par les attaques aériennes, par l'évacuation des élèves à la campagne et par le manque de manuels et de matériel scolaire que pratiquement plus personne ne pensait au développement des matières secondaires. 


\section{Conclusion} la zone française (ibid. : 217). Quand les trois zones de l'ouest s'unirent en 1949 pour former la République Fédérale d'Allemagne, presque tous les lycées modernes ainsi que ceux de mathématiques et de sciences naturelles se décidèrent pour l'anglais comme première langue étrangère et, à part dans les lycées de l'ancienne zone d'occupation française où l'on accepta des exceptions (Reinfried 2012: 179), le français devint la deuxième langue prototypique qu'elle est encore aujourd'hui. Naturellement, la prédominance de la langue anglaise à partir des années 1950 s'explique par la force politique et économique de la Grande Bretagne et surtout des États Unis. Néanmoins, le remplacement $d u$ français comme langue d'enseignement principale ne se serait pas fait aussi rapidement et radicalement en Allemagne de l'ouest si cette évolution n'avait eu les graves antécédents qu'elle a eus dans les années 1920 et 1930.

\section{BIBLIOGRAPHIE}

AEHLE, Wilhelm (1938). Die Anfänge des Unterrichts in der englischen Sprache, besonders auf den Ritterakademien. (Erziehungswissenschaftliche Studien, 7). Hamburg : Riegel.

ANON. (1921). « Zeitungsschau ». Deutsches Philologen-Blatt, 31, 376.

ANON. (1934). « Englisch oder Französisch - Inhalt oder Form? Eine rassenbiologische Erziehungsfrage ». Monatsschrift für höhere Schulen, 33, 172-174.

BENZE, Rudolf (1933). « Der Aufbau der deutschen Schule ». Volk im Werden, 1 (2), 29-40.

BOELITZ, Otto (1923). « Verfügungen der preußischen Unterrichtsverwaltung : Englisch als erste lebende Fremdsprache ». U II 157 U II W, U III D, U I. Deutsches Philologen-Blatt, 31, 141. 
BOHLEN, Adolf (1934). « Die nationalpolitische Linie im neusprachlichen Unterricht ». Neuphilologische Monatsschrift, 5, 1-20.

BÖLLING, Rainer (2010). Kleine Geschichte des Abiturs. Paderborn: Schöningh.

BOLLE, Wilhelm (1931). « Ein Vorschlag zur Vereinheitlichung des höheren Schulwesens ». Deutsches Philologen-Blatt, 39, 161-163.

BOLLE, Wilhelm (1934). « Die Sprachenfolge in der Kritik der nationalpolitischen Erziehung ». Monatsschrift für höhere Schulen, 33, 191-196.

CHRIST, Herbert (1983) : « Zur Geschichte des Französischunterrichts und der Französischlehrer ». In Anneliese Mannzmann (éd.), Geschichte der Unterrichtsfächer, vol. 1. München : Kösel, 94-117.

CHRIST, Herbert \& RANG, Hans-Joachim (éds.) (1985). Fremdsprachenunterricht unter staatlicher Verwaltung 1700 bis 1945. Eine Dokumentation amtlicher Richtlinien und Verordnungen. Vol. I. Einleitung und Orientierung ; Vol. IV. Neuere Fremdsprachen II ; Vol. VII. Der Fremdsprachenunterricht in Stundentafeln. Tübingen : Narr.

COLLISCHONN, Georg Adolf Otto (1922). « Die erste Fremdspra- che ». Deutsches Philologen-Blatt, $30,260-261$.

DEUTSCHBEIN, Max (1922). « Englisch als erste Fremdsprache ». Deutsches Philologen-Blatt, 30, 225-226.

ELIAS, Norbert ( $\left.{ }^{1} 1939 / 1997\right)$. Über den Prozeß der Zivilisation. Soziogenetische und psychogenetische Untersuchungen. Vol. 1. Wandlungen des Verhaltens in den weltlichen Oberschichten des Abendlandes. (Suhrkamp Taschenbuch Wissenschaft, 158.) Frankfurt a.M. : Suhrkamp 1997. (Première édition parue en 1939 à Basel).

ENGEL, Max (1927). « Pläne zur Vereinheitlichung des höheren Schulwesens in Berlin ». Deutsches Philologen-Blatt, 35, 766-769.

EULER, Arno (1977). Zur Entwicklung des Französischunterrichts seit 1945. In Michael Nerlich (éd.), Kritik der Frankreichforschung 1871-1975. (Argument, numéro spécial 13). Karlsruhe : Argument-Verlag, 216-233.

FÜHR, Christoph (21972). Zur Schulpolitik der Weimarer Republik. Die Zusammenarbeit von Reich und Ländern im Reichsschulausschuß (1919-1923) und im Ausschuß für das Unterrichtswesen (1924-1933). Darstellung und Quellen. Weinheim : Beltz. $\left.{ }^{1} 1970.\right)$

GRÄFER, Gustav (1939). « Der neusprachliche Unterricht nach den amtlichen Lehrplänen in ,Erziehung und Unterricht ». Die neueren Sprachen, 47, 223-234.

HAENICKE, Gunta (1982). Zur Geschichte des Faches Englisch in den Prüfungsordnungen für das Höhere Lehramt 1831-1942. (Augsburger I- \& I-Schriften, 4.) Augsburg : Universität.

HANF, Georg (1923). « Die deutsche Schule und das Französische. Ein Wort an unsere Neuphilologen ». Deutsches Philologen-Blatt, 31, 120-121.

HARTIG, Paul (1931). « Französisch muß wieder erste neuere Fremdsprache werden ». Sozialistische Monatshefte, 73, 339-345.

HASL, A. (1915/1916). « Neuphilologische Zeit- und Streitfragen ». Zeitschrift für französischen und englischen Unterricht, 14, 401-418, 15, 1-22, 96-124, 161-172.

HITLER, Adolf (1930/1941). Mein Kampf. Deux tomes dans un volume. München : Eher. 
HOMEYER, Alfred (1942). Die Neuordnung des höheren Schulwesens im Dritten Reich. Sammlung der wichtigsten diesbezüglichen Gesetze, Erlasse und Verfügungen seit Januar 1933. Mit 8 Nachträgen von 1941/42. Berlin : Klokow.

HÜBNER, Walter (1934). « Die Bildungsaufgaben der neueren Sprachen in der höheren Schule ». Monatsschrift für höhere Schulen, 33, 164-172.

HÜLLEN, Werner (2005). Kleine Geschichte des Sprachenlernens. Berlin : Schmidt.

JEISMANN, Karl-Ernst (1974). Das preußische Gymnasium in Staat und Gesellschaft. Die Entstehung des Gymnasiums als Schule des Staates und der Gebildeten, 1787-1817. (Industrielle Welt, 15.) Stuttgart : Klett.

JOHANNSEN, Hans M. (1929). « Völkerbund und Völkerversöhnung in der Schule ». Die Erziehung, 4, 449-455.

KLAPPER, Joseph (1935). « Der nationalpolitische Sinn des franzö-sischen Unterrichts ». Neuphilologische Monatsschrift, 6, 145-166.

KRÜPER, Adolf (1923). « Das Französische im Unterricht der deutschen Schule ». Deutsches Philologen-Blatt, 31, 143.

KUHFUSS, Walter (2014). Eine Kulturgeschichte des Französischunterrichts in der frühen Neuzeit. Französischlernen am Fürstenhof, auf dem Marktplatz und in der Schule in Deutschland. Göttingen : V\&R unipress.

LEHBERGER, Reiner (1986). Englischunterricht im Nationalsozialismus. Tübingen : Stauffenburg.

LEXIS, Wilhelm (éd.). « Die Reform des höheren Schulwesens in Preußen ». Halle a.S. : Verlag der Buchhandlung des Waisenhauses.

LUNDGREEN, Peter (1980/1981). Sozialgeschichte der deutschen Schule im Überblick. Vol. I. 1770-1918. (Kleine Vandenhoeck-Reihe, 1460). Vol. II. 1918-1980. (Kleine Vandenhoeck-Reihe, 1468.) Göttingen : Vandenhoeck \& Ruprecht.

MANGOLD, Wilhelm (1902). Der Unterricht im Französischen und Englischen. In Lexis (éd.), 191-226.

NAGEL, Anne C. (2012). Hitlers Bildungsreformer. Das Reichsministerium für Wissenschaft, Erziehung und Volksbildung 1934-1945. Frankfurt a.M. : Fischer Taschenbuch Verlag. OSTERMEIER, Christiane (2012). Die Sprachenfolge an den höheren Schulen in Preußen (1859-1931). Ein historischer Diskurs. Stutt- gart : Ibidem-Verlag.

REICHS- UND PREUSSISCHES MINISTERIUM für Wissenschaft, Erziehung und Volksbildung (1938). Erziehung und Unterricht in der Höheren Schule. Berlin : Weidmann.

REINFRIED, Marcus (1999). « Von der Realien- zur Kulturkunde. Frankreichkundliche Paradigmen als dialogische Konstrukte im deutschen Französischunterricht ». In Herwart Kemper, Siegfried Protz \& Detlef Zöllner (éds.). Schule, Bildung, Wissenschaft. Dia-Logik in der Vielfalt. Rudolstadt/ Jena : Hain, 199-234.

REINFRIED, Marcus (2001). « Französischunterricht im Dritten Reich: Rahmenbedingungen, Inhalte, Unterrichtsmethoden. » In Reinhard Dithmar \& Wolfgang Schmitz (éds.), Schule und Unterricht im Dritten Reich. (Interdisziplinäre Forschung und fächerverbindender Unterricht, 7). Ludwigsfelde : Ludwigsfelder Verlagshaus, 125-151.

REINFRIED, Marcus (2012). « Der Französischunterricht und seine quantitative Entwicklung an den deutschen Schulen (1965-2012) ». Französisch heute 43, 178-189. 
REINFRIED, Marcus (2013). «Die romanischen Schulsprachen im deutschen Schulwesen des Dritten Reichs : Sprachenpolitische Maßnahmen und bildungsideologische Diskurse ». In Friederike Klippel, Elisabeth Kolb \& Felicitas Sharp (éds.), Schulsprachenpolitik und fremdsprachliche Unterrichtspraxis. Historische Schlaglichter zwischen 1800 und 1989. (Münchener Arbeiten zur Fremdsprachen-Forschung, 26). Münster : Waxmann, 29-47.

REINFRIED, Marcus (2014). « European History of Romance language teaching ». In Christiane Fäcke (éd.), Manual of language acquisition. Berlin/Boston : de Gruyter, 255-273.

SANFTLEBEN, P. R. (1938). « Zur Schulreform ». Zeitschrift für den neusprachlichen Unterricht, $37,164-167$.

SCHNEIDER, Barbara (2000). Die Höhere Schule im Nationalsozialismus. Zur Ideologisierung von Bildung und Erziehung. Beiträge zur Historischen Bildungsforschung, 21. Köln : Böhlau.

SIEBERT, Theodor (1935). « Bedeutung und Wert der neueren Fremdsprachen für die höhere Schule im nationalsozialistischen Staat ». Neuphilologische Monatsschrift, 6, 273-294.

SIMON, Eduard (1928). « Das höhere Schulwesen Preußens 1928. Veränderungen im Bestande der höheren Lehranstalten 1927/28 ». Deutsches Philologen-Blatt, 36, 483-485.

SIMON, Eduard (1929). « Französisch oder Englisch als erste neuere Fremdsprache. Der gegenwärtige Stand an den preußischen höheren Lehranstalten und die durch die Sprachenfolge verursachte Mannigfaltigkeit der Schultypen ». Deutsches Philologen-Blatt, 37, 753-756.

SCHRÖDER, Konrad (1989). « Über Volkshaß und über den Gebrauch einer fremden Sprache. Zur historischen Dimension des Schulsprachenstreites Englisch-Französisch, unter besonderer Berücksichtigung der nach-Napoleonischen Zeit ». In Eberhard Kleinschmidt (éd.). Fremdsprachenunterricht zwischen Sprachenpolitik und Praxis. Festschrift für Herbert Christ zum 60. Geburtstag (Tübinger Beiträge zur Linguistik, 340), 58-70.

STÖLTING, A. (1922). « Französisch und Englisch auf den Realgymnasien ». Deutsches PhilologenBlatt, 30, 70-71.

WIESE, Oskar (1915). « Sollen wir die Kinder noch mit den Sprachen unserer Feinde quälen?». Blätter für höheres Schulwesen, 32, 209-211.

\section{NOTES}

1. L'exclusion politique et sociale de cette partie de la bourgeoisie était en Allemagne, comme le constate Elias (1997 : 113), plus habituelle qu'en France.

2. Il a néanmoins encore fallu attendre jusqu'en 1834, jusqu'à ce que la passation de l'Abitur devienne la condition sine qua non pour s'inscrire dans une université allemande (Bölling 2010 : 50).

3. Pendant la première période du bac (de 1788 à 1808), pendant laquelle la réglementation des examens n'était pas encore aussi strictement formulée et suivie par les écoles latines prussiennes, beaucoup de bacheliers rédigeaient déjà des dissertations en français, des versions ou des thèmes; vers le début du XX $\mathrm{XX}^{\mathrm{e}}$ siècle, il y avait dans les archives encore environ 300 travaux d'élèves (Kuhfuß 2014 : 591-596). Il y avait donc déjà un nombre relativement élevé d'élèves bien avancés en français. Mais à cause d'une francophobie née des guerres napoléoniennes, la Prusse, où une grande partie de la population était devenue nationaliste, a interdit l'enseignement du français dans ses écoles publiques entre 1816 et 1825 (à l'exception d'une de ses provinces, la Westphalie). Ensuite, jusqu'en 1837, l'enseignement du français a d'abord été facultatif dans la plupart des Gymnasien prussiens (Mangold 1902 : 191-192). 
1. Contrairement à Engel, Simon (1928: 485) indique pour la même année scolaire 28 nouvelles écoles commençant avec l'enseignement de l'anglais, ce qui correspondrait (selon les calculs de l'auteur M.R.) à 2,4\% de taux de croissance pour l'anglais première langue étrangère.

2. C'étaient le Mecklembourg-Schwerin, s'étendant le long d'une grande partie de la côte allemande de la mer Baltique, et le Mecklembourg-Strelitz, un petit territoire au sud-est du Mecklembourg-Schwerin.

3. La phrase originale est : «Aus dem Wesen des Völkerbundes ergibt sich, daß jeder Unterricht über ihn getragen sein muß [...] von der Einsicht, daß die Entwick-lung eines jeden Volkes gefördert wird durch die Zugehörigkeit zu einer umfas-senden Gemeinschaft aller Völker ».

4. Certains auteurs dans les revues des associations professionnelles des enseignants prétendent à l'époque qu'il y a des douzaines de différents types d'écoles. Dans un article, on peut même lire le nombre de 78 différentes formes d'écoles du secondaire réservées aux garçons (Aehle 1938 : 22).

5. L'accord des pays allemands a été élaboré et discuté à la fin de l'année 1931 lors de la neuvième réunion du Comité de l'éducation nationale des pays allemands à Berlin au Ministère de l'Intérieur (Führ $1972: 114$ ).

1. Le Ministère de l'éducation nationale allemand a officiellement été créé le $1^{\mathrm{er}}$ mai 1934. Il était fondé sur une union personnelle avec le Ministère de l'éducation prussien. La séparation des deux institutions devint ensuite trop compliquée pour les fonctionnaires, et à partir du $1^{\mathrm{er}}$ décembre 1934, on fusionna les deux ministères (Nagel 2012 : 66-67).

2. La dictature nationale-socialiste ne simplifia pas les modifications scolaires, comme on pourrait s'imaginer, elle les compliqua. Hitler n'osait pas dissoudre les nombreux ministères de l'éducation régionaux (chaque pays allemand continuait à disposer de son propre ministère) parce que les ministres généralement nommés en 1933 étaient souvent des anciens compagnons qui avaient déjà intensément milité au sein du parti national-socialiste, presque dès ses débuts, et devaient maintenant être "récompensés "; il ne voulait pas les frustrer. La fidélité absolue de ces paladins indéfectibles était une base importante du pouvoir d'Hitler. De plus, l'organisation du NSDAP (parti national-socialiste) prenait au cours du Troisième Reich un nombre croissant de décisions politiques dans tous les domaines de la vie quotidienne et entrait en concurrence avec les institutions de l'État. Pour le Ministère national de l'éducation, il devenait de plus en plus nécessaire de s'accorder avec certains spécialistes du NSDAP. Beaucoup de décisions éducatives dépendaient désormais de l'acceptation d'institutions externes à l'administration traditionnelle comme par exemple la Hitlerjugend, association pour la jeunesse associée au NSDAP (Nagel 2012: 85-90, 196).

3. La phrase originale et complète est : «Dem romanischen Menschen und seinem Volkstum mit all seinen für uns so gefährlichen Fehlentwicklungen wird immer in stärkerem Maße eine negativ-warnende, als eine positiv erziehende Wirkung auf unsere Jugend zuzuerkennen sein, und diese Tatsache allein weist dem Französischen eine erkenntnisreiche, aber durchaus zweitrangige Stellung im Bildungsplan der deutschen Schulen zu ».

4. La phrase originale est: «[...] Demgegenüber verlangt die völkisch verantwortungsvolle Erziehung auch auf geistigem Gebiete, daß alle Bildungsziele und Bildungsstoffe nur auf den Kreis des eigenen Rassen- und Volkstums bezogen werden und daß alles vermieden wird, was geeignet ist, den jungen Menschen durch vorzeitiges und übermäßiges Herantragen wesensfremder Dinge von seinem völkischen Lebenskreise abzuziehen ».

5. La phrase originale est : «Wir Deutschen haben unsere Begabungsstärke nicht in erster Linie auf dem Gebiet der Formgebung und der Sprachgewandtheit wie etwa der Jude oder der Südeuropäer ».

6. La phrase originale et complète est : "Das Englische ist die Anfangs- und Hauptsprache der neuen Oberschule, da es die Sprache eines uns rassisch verwandten Volkes ist, das aus großer 
politischer Begabung weltpolitische Leistungen vollbracht und seine Sprache zur Verkehrssprache der Weltwirtschaft gemacht hat ».

1. Certains passages contre langue et culture dans l'autobiographie Mein Kampf (Hitler 1941:58, 465-466, 695-699, 704-705, 766-767) sont extrêmement polémiques et grotesques. Hitler avait une aversion contre la langue française (inutile dans la vie pratique et inutile à l'entrainement de la pensée logique), contre le peuple français («l'ennemi mortel du peuple allemand»), contre la « race française » (« pervertie par les nègres et les Juifs»), contre l'État français (il devra être détruit par les Allemands) et contre la culture française (« minablement faible »).

\section{RÉSUMÉS}

Vers 1810, les prestigieux Gymnasien ont été institutionnalisés en Allemagne. Ils ont été très influencés par le néo-humanisme qui y atteignit son apogée dans la première moitié du XIX siècle. Le français langue étrangère avait peu de valeur éducative dans ce contexte, mais il a été enseigné en Prusse à partir de 1837 comme matière obligatoire, mais secondaire, et pour de seules raisons utilitaires. Vers la fin du XIX ${ }^{\mathrm{e}}$ siècle, il est devenu de plus en plus important dans les Realgymnasien et Oberrealschulen qui ont commencé à faire concurrence aux Gymnasien. L'anglais s'est parallèlement établi comme deuxième langue vivante.

La Première Guerre mondiale et l'occupation du bassin de la Ruhr en 1923 ont provoqué des aversions contre les Français et leur langue dans une grande partie de la population allemande. $\mathrm{Au}$ cours des années vingt, l'offre des langues fut flexibilisée, et à la fin de la décennie, les chiffres du français et de l'anglais comme première langue sont parvenus à un niveau d'égalité. En 1931, Adolf Grimme, ministre de l'éducation en Prusse, essaya de rétablir l'ancienne importance du français. Mais la réforme scolaire des nazis, réglée par des décrets à partir de 1936, prévoyait une position privilégiée de l'anglais et une position marginalisée du français, qui fut ravalé, dans la plupart des cas, au rang de troisième langue. Cette décision fut prise pour des raisons idéologiques.

Around the year 1810, the prestigious Gymnasien were institutionalized in Germany. They were very influenced by Neohumanism which reached its climax in the first half of the $19^{\text {th }}$ century. In this context, the French foreign language had few educational values, but it was taught since 1837 as a compulsory minor subject solely for considerations as to its usefulness. Towards the end of the $19^{\text {th }}$ century, it has become more and more important in the Realgymnasien and Oberrealschulen which began to compete with the Gymnasien. The English foreign language has simultaneously been established as a second living language.

\section{INDEX}

Keywords : Foreign language teaching. 19th century. 20th century. Modern languages in Germany. Language choice. Language policy. Nationalism. National Socialism.

Mots-clés : enseignement des langues étrangères, XIXe siècle, XXe siècle, langues modernes en Allemagne, choix des langues, politique des langues, nationalisme, national-socialisme 
AUTEUR

\section{MARCUS REINFRIED}

Friedrich-Schiller-Universität Jena

marcus.reinfried@uni-jena.de 